УДК 532.536

\title{
ДИНАМІКА РОЗГІННОЇ ТЕЧІЇ В МІКРОЦИЛІНДРІ, ЩО ПОЧИНАЄ РАПТОВО ОБЕРТАТИСЯ
}

Авраменко А.О., чл.-кор. НАН України, Тирінов А.І., канд. техн. наук, Дмитренко Н.П., канд. техн. наук, Кравчук О.В.

Інститут технічної теплофізики НАН Украӥни, вул. Желябова 2а, м. Київ, 03068, Украӥна

В статті викладені результати теоретичного дослідження розгінної течії нестисливої рідини з раптовим початком обертання мікроціліндра. Задача вирішувалася за допомогою двох аналітичних підходів - методу Фур'є і аналізу симетрії. В ході аналітичного рішення отримано вирази для обчислення профілю швидкості, коефіцієнта тертя та крутного моменту.
В статье изложены результаты теоретического исследования разгонного течения несжимаемой жидкости с внезапным началом вращения микроцилиндра. Задача решалась с помощью двух аналитических подходов - метода Фурье и анализа симметрии. В ходе аналитического решения получены выражения для вычисления профиля скорости, коэффициента трения и крутящего момента.
The article presents the results of theoretical research accelerating flow of an incompressible fluid with a sudden onset microcylinder rotation. The problem was solved with the help of two analytical approaches - Fourier analysis method and symmetry. In the course of the analytical solutions the expressions for the calculation of the velocity profile, the coefficient of friction and torque are obtained.

Бібл. 12, рис. 1.

Ключові слова: гідродинаміка, розгінний потік мікроциліндр, коефіцієнт тертя, профіль швидкості.

$A, a, b-$ константи;

$L$ - довжина вільного пробігу молекули;

$\widetilde{r}$ - радіальна координата;

$\widetilde{t}$ - час;

$\tilde{u}$ - колова швидкість

\section{Введення}

Науковий i практичний інтерес до мікроканальних потоків постійно зростає в останні десятиліття в зв'язку з розвитком різних мікропристроїв та мікроелектромеханічних систем. В мікропристроях, вимірювання та моделювання величин проходить в мікромасштабах 3 характерною довжиною в діапазоні мікрометрів. Ефекти розрідження мікропотоків характеризуються числом Кнудсена $(\mathrm{Kn})$. Для $\mathrm{Kn} \leq 10^{-3}$ потік все ще можна змоделювати за допомогою рівнянь Нав'є-Стокса, які замикаються умовами прилипання. При $10^{-3} \leq \mathrm{Kn} \leq 10^{-1}$ виникає так званий режим потоку з проковзуванням [1]. Ці межі іще не чітко визначені до сих пір. Режим переходу потоку виникає при $10^{-1} \leq \mathrm{Kn} \leq 10$, де припущення континуума не діє і застосування такого методу моделювання як Монте-Карло чи рівнянь $\alpha$ - коефіцієнт в'язкого ковзання;

$\mu$ - динамічний коефіцієнт в'язкості;

$v$ - коефіцієнт кінематичної в'язкості;

$\rho-$ густина.
Барнета [2] є можливим.

В даний час цілий ряд методів було розроблено, для моделювання мікро- та нанопотоків, що включають різні довжини і час. Вони можуть бути розділені на наступні групи.

Для мікромасштабного діапазону близько 10 нм i 1 нс, використовуються методи молекулярної динаміки, які беруть до уваги тільки консервативний потенціал міжмолекулярного зв'язку і невалентні сили [3, 4]. В мезомасштабному діапазоні приблизно від 10 нм до 1 нм і від 1 нс до 10 мс, використовують метод динаміки дисипативних частинок, який включає в себе не тільки сили відштовхування, а i дисипативні і стохастичні взаємодії. Метод граток Больцмана, який оснований на решітчастому рівнянні Больцмана [5,6] також може бути використаних тут. 
Розгінну течію в трубі, де перепад тиску не залежить від часу, було досліджено спочатку для початкової нерухомої рідині. Перше точне рішення цієї задачі було отримано в [7] при розгінній течії в круглій трубі. Розгінний потік в кільцевому каналі був вивчений в [8]. Зворотне явище, а саме, зупинка потоку в трубі з падінням тиску, що раптово зникає було розглянуто в [9]. Розгінний потік в круглій трубі і між паралельними пластинам, простір між якими заповнений пористим середовищем, яке рівномірно насичене рідиною було досліджено в [10].

В даній роботі розглянуто розгінну течію в мікроциліндрі, який починає раптово обертатися 3 постійною швидкістю. Розглянута нестислива рідина. Було застосовано два методи - метод методу Фур'є та метод симетрії (групи Лі).

\section{Математична модель}

У момент часу $\tilde{t}=0$ швидкість внутрішнього циліндру збільшується миттєво від нуля до $\widetilde{u}_{0}$. Рух такого потоку описується наступним рівнянням

$$
\frac{\partial \tilde{u}}{\partial \widetilde{t}}=v\left(\frac{\partial^{2} \tilde{u}}{\partial \widetilde{r}^{2}}+\frac{1}{\widetilde{r}} \frac{\partial \tilde{u}}{\partial \widetilde{r}}-\frac{\tilde{u}}{\widetilde{r}^{2}}\right)
$$

Граничні та початкові умови мають наступний вигляд:

$\tilde{u}=0$ для всіх $\widetilde{r}$ при $\tilde{t} \leq 0$

$\widetilde{u}=0$ при $\widetilde{r}=0$;

$\widetilde{u}=\widetilde{u}_{0}-\alpha L \frac{\partial \tilde{u}}{\partial \widetilde{r}}$ для $\widetilde{r}=\widetilde{r}_{0}$ при $\tilde{t}>0$.

Для подальших розрахунків зручно скористатися наступними безрозмірними величинами:

$$
r=\frac{\widetilde{r}}{\widetilde{r}_{0}}, \quad u=\frac{\widetilde{u}}{\widetilde{u}_{0}}, \quad t=\frac{\widetilde{t} \mathrm{v}}{{\widetilde{r_{0}}}^{2}} .
$$

Враховуючи безрозмірні величини (5) та умови (3) i (4) рівняння (2) прийме наступну форму:

$\frac{\partial u}{\partial t}=\frac{\partial^{2} u}{\partial r^{2}}+\frac{1}{r} \frac{\partial u}{\partial r}-\frac{u}{r^{2}}$

$u=0$ для всіх $r$ при $t \leq 0$

$u=0$ для $r=0$

$u=1-\mathrm{Kn} \frac{\partial u}{\partial r}$ для $r=1$ при $t>0$,

$\mathrm{Kn}=\frac{\alpha L}{\widetilde{r}_{0}}$
- число Кнудсена.

Для стаціонарної течії рівняння (6) - (8) трансформуються в

$\frac{\partial^{2} u}{\partial r^{2}}+\frac{1}{r} \frac{\partial u}{\partial r}-\frac{u}{r^{2}}=0$

$u=0$ при $r=0$

$u=1-\mathrm{Kn} \frac{\partial u}{\partial r}$ при $r=1$

Рішення рівняння (10) з граничними умовами (11) та (12) має просту форму

$u=\frac{r}{1+\mathrm{Kn}}$.

Для випадку течії без проковзування $(\mathrm{Kn}=0)$ рівняння (13) буде мати класичний вигляд [11] $u=r$.

\section{Memod Фур'є}

Враховуючи умову (8), будемо шукати рішення у наступному вигляді

$u(t, r)=A r+\sum_{n=1}^{\infty} R_{n}(r) T_{n}(t)$.

3 урахуванням цієї умови, маємо

$A=\frac{1}{1+\mathrm{Kn}}$.

Підстановка (15) в (6) дає

$\sum_{n=1}^{\infty}\left[T_{n}\left(R_{n}^{\prime \prime}+\frac{1}{r} R_{n}^{\prime}-\frac{1}{r^{2}} R_{n}\right)-T_{n} R_{n}^{\prime}\right]=0$.

Розділяючи змінні, отримуємо

$\frac{R_{n}^{\prime \prime}+\frac{1}{r} R_{n}^{\prime}-\frac{1}{r^{2}} R_{n}}{R_{n}}=\frac{T_{n}^{\prime}}{T_{n}}=-z_{n}^{2}$.

Звідси маємо

$r^{2} R_{n}^{\prime \prime}+r R_{n}^{\prime}+\left[\left(r z_{n}\right)^{2}-1\right] R_{n}=0$.

Рішенням цього рівняння, з урахуванням граничних умов, $\epsilon$

$R_{n}=J_{1}\left(z_{n} r\right)$,

де $J_{1}-$ функції Бесселя першого роду першого порядку, $z_{n}$ - корені трансцендентного рівняння

$\left(R_{n}+\mathrm{Kn} R_{n}^{\prime}\right)_{r=1}=0$.

Із (17) також маємо

$T_{n}^{\prime}+z_{n}^{2} T_{n}=0$.

Звідки

$T_{n}=c_{n} e^{-z_{n}^{2} t}$. 
Враховуючи початкову умову $t=0$ та властивості ортогональності знаходимо

$c_{n}=-A \frac{\int_{0}^{1} r R_{n} r d r}{\int_{0}^{1} R_{n}^{2} r d r}$.

Тоді кінцевий вираз для швидкості має наступну форму

$$
\begin{aligned}
& u(t, r)=\frac{r}{1+\mathrm{Kn}}+! \\
& +2 \sum_{n=1}^{\infty} \frac{J_{1}\left(z_{n} r\right)}{z_{n}(1+2 \mathrm{Kn}) J_{0}\left(z_{n}\right)+\left(1-\mathrm{Kn}-z_{n}^{2} \mathrm{Kn}\right) J_{1}\left(z_{n}\right)} \exp \left(-z_{n}^{2} t\right),
\end{aligned}
$$

де $J_{0}-$ функції Бесселя першого роду нульового порядку.

При $\mathrm{Kn} \rightarrow 0$ вираз (25) трансформується до

$u(t, r)=r+2 \sum_{n=1}^{\infty} \frac{J_{1}\left(z_{n} r\right)}{z_{n} J_{0}\left(z_{n}\right)} \exp \left(-z_{n}^{2} t\right)$,

де

$$
J_{1}\left(z_{n}\right)=0 \quad(n=1,2, \ldots) .
$$

\section{Симетричне рішення}

Для автомодельних перетворень рівняння (6) необхідно знайти симетрії (групи Лі) цього рівняння. Симетрії рівняння (6) можна описати за допомогою інфінітезимального генератора [12]

$$
q=\xi_{1} \frac{\partial}{\partial t}+\xi_{2} \frac{\partial}{\partial r}+\phi \frac{\partial}{\partial u},
$$

де $\xi 1, \xi 2, \phi-$ невідомі коефіцієнти. Ці коефіцієнти знаходяться із наступної умови

$\operatorname{pr}^{(2)} q(\Delta)=0$,

де $p^{(2)} q(\Delta)$ - друга пролонгація інфінітезимального генератора (28), оператор $\Delta$ означає часткове диференціювання рівняння (6).

Друга пролонгація інфінітезимального генератора (28) побудована у відповідності з наступним виразом

$$
p r^{(2)} q=q+\phi^{t} \frac{\partial}{\partial u_{t}}+\phi^{r} \frac{\partial}{\partial u_{r}}+\phi^{r r} \frac{\partial}{\partial u_{r r}},
$$

де індекси функції $u$ являють собою часткові похідні по відповідним змінним. Коефіцієнти $\phi^{t}$, $\phi^{r}, \phi^{r r} \epsilon$ функціями $\xi_{1}, \xi_{2}, \phi$ та $u$ їхніми похідними по $t$ та $r$.

Застосування оператора $p r^{(2)} q$ до рівняння (6) дає вираз, який вміщає одночлени 3 різними комбінаціями функції $u$. В результаті ці члени приводять до системи диференціальних рівнянь відповідно до коефіцієнтів $\xi_{1}, \xi_{2}, \phi$. Розв'язок цієї системи дає шість симетрій (перетворень Лі). Ці алгебри Лі $\epsilon$

$$
q_{1}=\frac{\partial}{\partial t}
$$

$$
q_{2}=u \frac{\partial}{\partial u}
$$

$$
q_{3}=2 t \frac{\partial}{\partial t}+r \frac{\partial}{\partial r}
$$

$$
q_{4}=4 t^{2} \frac{\partial}{\partial t}+4 t r \frac{\partial}{\partial r}+u\left(4 t+r^{2}\right) \frac{\partial}{\partial u}
$$

$$
q_{5}=\exp \left(a^{2} t\right) J_{1}(a r) \frac{\partial}{\partial u}
$$

$$
q_{6}=\exp \left(b^{2} t\right) Y_{1}(b r) \frac{\partial}{\partial u},
$$

де $Y_{1}$ - функція Бесселя другого роду першого порядку.

Використовуючи перетворення Лі, можна понизити кількість змінних і автомодельні форми рівняння (6). Для цього зручно використати комбінацію масштабованих трансформацій

$q_{2,3}=C_{2} u \frac{\partial}{\partial u}+C_{3}\left(2 t \frac{\partial}{\partial t}+r \frac{\partial}{\partial r}\right)$,

де $C_{2}$ i $C_{3}-$ константи.

Автомодельні змінні можна знайти за допомогою перетворення $q_{3}$, яке генерує наступне рівняння

$2 t \frac{\partial \eta}{\partial t}+r \frac{\partial \eta}{\partial r}=0$

Розв' язком рівняння (38) є

$\eta=\frac{r}{\sqrt{v t}}$.

Щоб отримати автомодельну форму невідомої функції потрібно вибрати параметричну змінну. Зазвичай вона обирається такою, похідна по якій має найменший порядок. Тобто із (38) отримуємо $C_{3} 2 t \frac{\partial F}{\partial t}+C_{2} u \frac{\partial F}{\partial u}=0$.

Розв’ язком (40) буде

$u=t^{\frac{C_{2}}{2 C_{3}}} \Phi$. 
$C_{2}$ та $C_{3}$ - довільні константи. Далі наведемо автомодельну форму невідомої функції в такому вигляді

$u=t^{g} \Phi(\eta)$,

де $g$ - довільний коефіцієнт.

Підставивши (42) в (6) маємо

$\frac{d^{2} \Phi}{d \eta^{2}}+\left(\frac{\eta}{2}+\frac{1}{\eta}\right) \frac{d \Phi}{d \eta}-\left(g+\frac{1}{\eta^{2}}\right) \Phi=0$.

Умови (7) та (8) трансформуються в

$$
\begin{array}{lll}
\Phi=0 & \text { для } & \eta=0, \\
\Phi=\frac{1}{t^{g}}-\frac{\text { Kn }}{\sqrt{t}} \frac{d \Phi}{d \eta} & \text { для } & \eta=\frac{1}{\sqrt{t}} .
\end{array}
$$

Для вирішення рівняння (43) перетворимо його до стандартної (класичної) форми. Для цього необхідно включити наступну заміну

$\Phi=\eta \Psi(\xi(\eta))$,

де

$\xi(\eta)=-\frac{\eta^{2}}{4}$.

Використовуючи рівняння (46) i (47) можна трансформувати рівняння (43) до

$\xi \frac{d^{2} \Psi}{d \xi^{2}}+(2-\xi) \frac{d \Psi}{d \xi}-\left(\frac{1}{2}-g\right) \Psi=0$.

Рівняння (48) є класичним рівнянням Куммеpa і рішенням цього рівняння $\epsilon$

$\Psi=C_{11} F_{1}\left(\frac{1}{2}-g, 2, \xi\right)+C_{2} M\left(\frac{1}{2}-g, 2, \xi\right)$,

де $C_{1}$ та $C_{2}$ - константи інтегрування, $F_{1}-$ функція Куммера (гіпергеометрична функція), $M$ - другий лінійний незалежний розв'язок, який можна виразити через функцію Куммера.

Підставляючи рівняння (49) в (46) і беручи до уваги (47) з використанням граничних умов (44) i (45), ми отримаємо

$$
\Phi=\frac{8 t^{\frac{3}{2}-g} \eta_{1} F_{1}\left(\frac{1}{2}-g, 2,-\frac{\eta^{2}}{4}\right)}{8 t(1+\mathrm{Kn})_{1} F_{1}\left(\frac{1}{2}-g, 2,-\frac{1}{4 t}\right)+(2 g-1) \mathrm{Kn}_{1} F_{1}\left(\frac{3}{2}-g, 3,-\frac{1}{4 t}\right)},
$$

Після підстановки (50) в (42) отримаємо розподіл швидкості в вигляді

$$
u(t, r)=\frac{8 r t{ }_{1} F_{1}\left(\frac{1}{2}-g, 2,-\frac{r^{2}}{4 t}\right)}{8 t(1+\mathrm{Kn})_{1} F_{1}\left(\frac{1}{2}-g, 2,-\frac{1}{4 t}\right)+(2 g-1) \mathrm{Kn}_{1} F_{1}\left(\frac{3}{2}-g, 3,-\frac{1}{4 t}\right)} .
$$

Якщо ми врахуємо умову $t \rightarrow \infty$, то (51) перетвориться на (12).

При $\mathrm{Kn} \rightarrow 0$ рівняння(51) скоротиться до

$$
u(t, r)=\frac{r_{1} F_{1}\left(\frac{1}{2}-g, 2,-\frac{r^{2}}{4 t}\right)}{{ }_{1} F_{1}\left(\frac{1}{2}-g, 2,-\frac{1}{4 t}\right)} .
$$

\section{Результати дослідження}

На основі отриманих профілів швидкості можливо розрахувати динаміку поведінки поверхневого тертя $\tau_{w}$, використовуючи наступну формулу

$\tau_{w}=\left.\mu\left(\frac{\partial \widetilde{u}}{\partial \widetilde{r}}-\frac{\widetilde{u}}{\widetilde{r}}\right)\right|_{\widetilde{r}=\widetilde{r}_{0}}$.

Рівняння (53) перепишемо у вигляді

$c_{f}=\left.\frac{4}{\operatorname{Re}}\left(\frac{\partial u}{\partial r}-\frac{u}{r}\right)\right|_{r=1}$,

де

$c_{f}=\frac{2 \tau_{w}}{\rho \tilde{u}_{0}^{2}}$

- коефіцієнт тертя.

$\operatorname{Re}=\frac{\rho \widetilde{u}_{0} 2 \widetilde{r}_{0}}{\mu}$

- число Рейнольдса.

Використовуючи (25) вираз (54) перетрансформується в

$\frac{c_{f} \operatorname{Re}}{4}=2 \sum_{n=1}^{\infty} \frac{J_{0}\left(z_{n}\right)-2 J_{1}\left(z_{n}\right)}{z_{n}(1+2 \mathrm{Kn}) J_{0}\left(z_{n}\right)+\left(1-\mathrm{Kn}-z_{n}^{2} \mathrm{Kn}\right) J_{1}\left(z_{n}\right)} \exp \left(-z_{n}^{2} t\right)$.

Якщо ми застосуємо групи симетрії (46) то отримаємо

$\frac{c_{f} \mathrm{Re}}{4}=\frac{(2 g-1)_{1} F_{1}\left(\frac{3}{2}-g, 3,-\frac{1}{4 t}\right)}{8 t(1+\mathrm{Kn})_{1} F_{1}\left(\frac{1}{2}-g, 2,-\frac{1}{4 t}\right)+(2 g-1) \mathrm{Kn}_{1} F_{1}\left(\frac{3}{2}-g, 3,-\frac{1}{4 t}\right)}$

Розподіли коефіцієнта тертя в залежності від часу, отримані на основі виразів (57) та (58) наведено на рис. 1. Пунктирна лінія відповідає результатам отриманим на основі (57), а суцільні лінії відповідають виразу (58) для різних значень параметру $g$.

В ході аналізу результатів розрахунку виявилося, що в початковий момент часу коефіцієнт тертя більший для потоку, що проковзує. Однак в процесі розвитку потоку значення коефіцієнта тертя для потоку, що проковзує, стає меншим в 
порівнянні з потоком, що прилипає до стінки. Це під час розвитку течії на твердій поверхні для поможна пояснити зростанням скачка швидкості току, що прилипає.

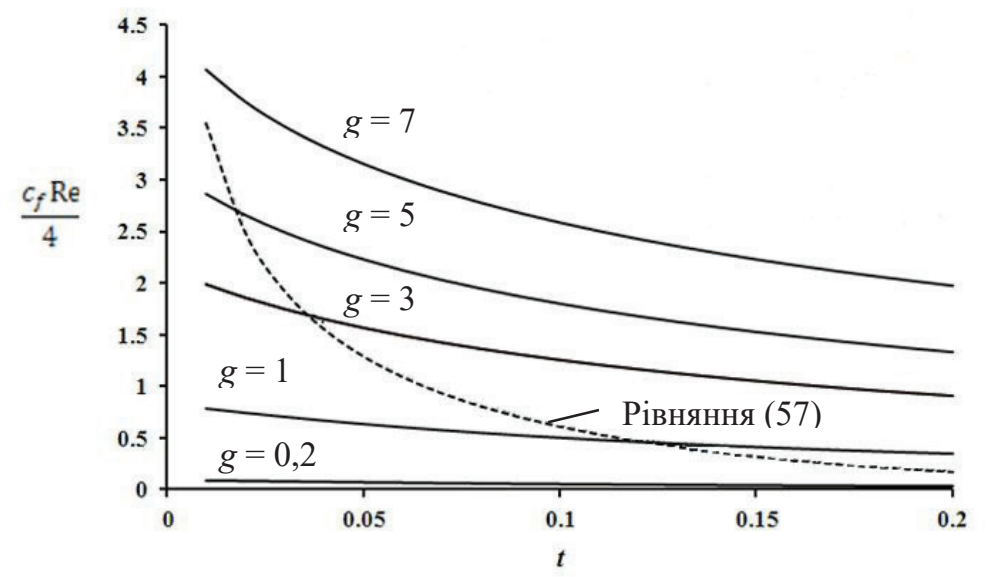

Рис. 1. Залежність коефіцієнта тертя від часу при $\mathrm{Kn}=0,1$.

Якщо помножити коефіцієнт поверхневого тертя на радіус циліндра і довжину його кола, то отримаємо величину моменту, який повинен бути прикладений до циліндра, щоб підтримувати його обертання з постійною швидкістю. В нашому випадку цей крутний момент визначається із

$$
L=4 \mu \pi \widetilde{u}_{0} \widetilde{r}_{0} \sum_{n=1}^{\infty} \frac{J_{0}\left(z_{n}\right)-2 J_{1}\left(z_{n}\right)}{z_{n}(1+2 \mathrm{Kn}) J_{0}\left(z_{n}\right)+\left(1-\mathrm{Kn}-z_{n}^{2} \mathrm{Kn}\right) J_{1}\left(z_{n}\right)} \exp \left(-z_{n}^{2} t\right) .
$$

Для задачі симетрії

$$
L=\frac{\mu \pi \tilde{u}_{0} \widetilde{r}(2 g-1)_{1} F_{1}\left(\frac{3}{2}-g, 3,-\frac{1}{4 t}\right)}{2 t(1+\mathrm{Kn})_{1} F_{1}\left(\frac{1}{2}-g, 2,-\frac{1}{4 t}\right)+(2 g-1) \mathrm{Kn}_{1} F_{1}\left(\frac{3}{2}-g, 3,-\frac{1}{4 t}\right)} .
$$

Як можна бачити, значення зазначеного крутного моменту, наближається до нуля із зростанням часу.

\section{Висновки}

Проведено аналітичний аналіз розгінного потоку в мікроциліндрі, що починає раптово обертатися. Аналітичні рішення отримані на основі двох математичних методів: Фур’є і груп симетрії. Метод Фур'є дав розв'язок у вигляді нескінченного ряду, а методика симетрії дала рішення у вигляді кінцевого рівняння.

Коефіцієнт тертя зменшується 3 часом від нескінченності при $t=0$ до нуля, якій відповідає стаціонарній течії. У початковий момент часу коефіцієнт тертя вище для потоку з проковзуванням. Однак в процесі розвитку потоку значення коефіцієнта тертя для потоку з прилипанням на стінці стає більшим, ніж для потоку з ковзанням.

\section{ЛІТЕРАТУРА}

1. Gad-el-Hak M. The fluid mechanics of microdevices // J. Fluids Engineering. - 1999. - V. 121 - P. 5-33.

2. Bird G.A. Molecular Gas Dynamics and the Direct Simulation of Gas Flows. - Oxford University Press: $1994 .-$ p. 458.

3. Haile J.M. Molecular dynamics simulation. New York Wiley and sons. - 1992. - p. 481.

4. G. Karniadakis, A. Beskok, Aluru N. Microflows and Nanoflows Fundamentals and Simulation. - New York: Springer. - 1965. - p. 818.

5. Wylie B.J.N. Application of two-dimensional cellular automaton lattice-gas models to the simulation of hydrodynamics. - University of Edinburgh. - 1990. - p. 114.

6. Maxwell J.B. Lattice Boltzmann methods for interfacial wave modelling. - University of 
Edinburgh. - 1997. - p. 238.

7. Szymansky F. Quelques solution exactes des équations de l'hydrodynamique de fluide visqueux dans le cas d'un tube cylindrique // J. Math. Pures Appl. -1932 V.97, - №.11, - P. 67-107.

8. Müller W. Zum Problem der Anlaufströmung einer Flüssigkeit im geraden Rohr mit Kreisringund Kreisquerschnitt // ZAMM. - 1936. - №.16, - P. 227-238.

9. Gerberts W. Zur instationären, laminaren
Strömung einer inkompressiblen zähen Flüssigkeit in kreiszylindrischen Rohren // Z. angew. Physik. 1951. - V.3, - P. 267-271.

10. Avramenko A.A., Kuznetsov A.V. Start-up flow in a channel or pipe occupied by a fluid-saturated porous medium // J. Porous Media. - 2009. - V.12, №. 4. - P. 361-367.

11. Schlichting H., Gersten K. Boundary Layer Theory, 8th ed. Berlin. Springer: - 2000. p. 799.

12. Olver P. Applications of Lie Groups to Differential Equations. Berlin. Springer: - 2000. p. 513. 


\section{DYNAMICS ACCELERATING FLOW IN MICROCYLINDER THAT START SUDDENLY ROTATES}

\section{Avramenko A.O., Tyrinov A.I., Dmitrenko N.P., Kravchuk O.V.}

Institute of Engineering Thermophysics of the National Academy of Sciences of Ukraine, st. Zhelyabova, 2a, Kyiv, 03680 Ukraine

An analytical analysis startup flow inside the microcylinder when it starts suddenly rotating is analyzed. Analytical solutions are obtained using of two mathematical methods: Fourier and symmetry groups. Fourier method of solution given in the form of an infinite series, and a symmetry method gives solution in closed form.

The results of the investigation show that the coefficient of friction decreases with time from infinity at $t=0$ to zero, which corresponds to a stationary flow. At the initial time coefficient of friction is higher for the slip flow. At the initial time the coefficient of friction for the slip flow is greater than for the flow without sliding.

Key words: hydrodynamics, startup flow, microcylinder, friction coefficient, velocity profile.

References 12, figure 1.

1. Gad-el-Hak M. The fluid mechanics of microdevices // J. Fluids Engineering. - 1999. - V. 121 - P. 5-33.

2. Bird G.A. Molecular Gas Dynamics and the Direct Simulation of Gas Flows. - Oxford University
Press: 1994. - p. 458.

3. Haile J.M. Molecular dynamics simulation. New York Wiley and sons. - 1992. - p. 481.

4. G. Karniadakis, A. Beskok, Aluru N. Microflows and Nanoflows Fundamentals and Simulation.New York: Springer. - 1965. - p. 818.

5. Wylie B.J.N. Application of two-dimensional cellular automaton lattice-gas models to the simulation of hydrodynamics. - University of Edinburgh. - 1990. - p. 114.

6. Maxwell J.B. Lattice Boltzmann methods for interfacial wave modelling. - University of Edinburgh. - 1997. - p. 238.

7. Szymansky F. Quelques solution exactes des équations de l'hydrodynamique de fluide visqueux dans le cas d'un tube cylindrique // J. Math. Pures Appl. -1932 V.97, - №.11, - P. 67-107.

8. Müller $W$. Zum Problem der Anlaufströmung einer Flüssigkeit im geraden Rohr mit Kreisringund Kreisquerschnitt // ZAMM. - 1936. - №.16, - P. 227-238.

9. Gerberts $W$. Zur instationären, laminaren Strömung einer inkompressiblen zähen Flüssigkeit in kreiszylindrischen Rohren // Z. angew. Physik. 1951. - V.3, - P. 267-271.

10. Avramenko A.A., Kuznetsov A.V. Start-up flow in a channel or pipe occupied by a fluid-saturated porous medium // J. Porous Media. - 2009. - V.12, №. 4. - P. 361-367.

11. Schlichting H., Gersten K. Boundary Layer Theory, 8th ed. Berlin. Springer: - 2000. p. 799.

12. Olver P. Applications of Lie Groups to Differential Equations. Berlin. Springer:-2000. p. 513.

Получено 15.08.2016 Received 15.08.2016 